\title{
Unusual left ventricular wall motion and a loud added sound during the isovolumic relaxation period in a patient with hypertensive heart disease
}

\author{
Yuichiro Mishiro, Takashi Oki, Nobuo Fukuda
}

\begin{abstract}
A 69 year old woman with hypertensive heart disease had a loud added sound which coincided with a sudden interruption of the early diastolic motion of the left ventricular posterior wall, as visualised by $M$ mode echocardiography, and came just before early diastolic transmitral flow, as measured by a pulsed Doppler echocardiogram. Early diastolic motion velocity from the base to the middle of the posterior wall, assessed by pulsed Doppler tissue imaging, was markedly high and sharp, and its peak coincided with the sound. A notch, similar to that in the posterior wall motion, occurred in the left ventricular pressure curve during early diastole. No intraventricular flow signal was detected during the isovolumic relaxation period, as measured by pulsed and colour Doppler imaging. The added sound was probably produced by impact between the dilated heart, with a relaxation abnormality, and the extracardiac structures during the isovolumic relaxation period.
\end{abstract}

(Heart 1997;77:290-292)

Apex
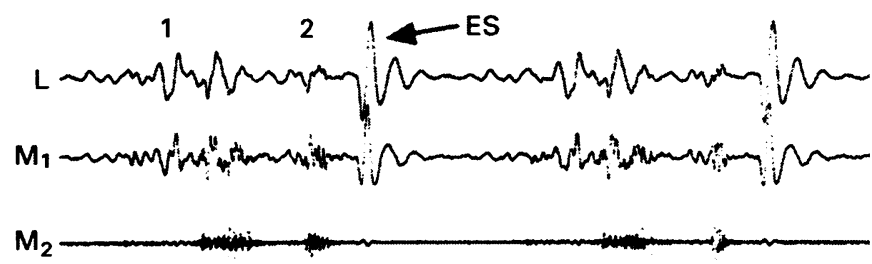

3L
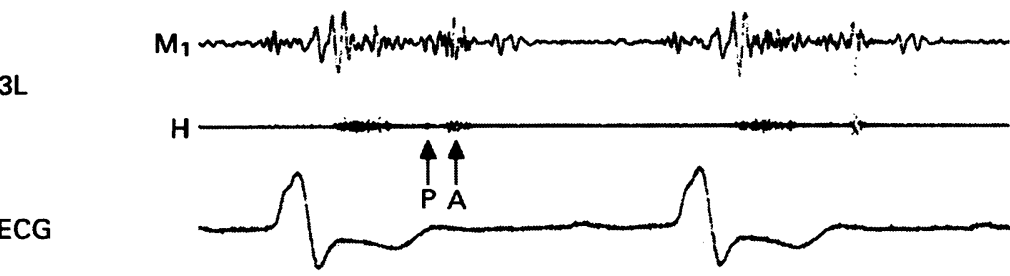

Figure 1 Phonocardiogram: the first heart sound (1) is decreased, and the second heart sound (2) indicates reversed splitting; a loud low pitched extra sound (ES) is observed at the cardiac apex during early diastole. $L, M 1, M 2$, and $H$ : low, medium low, medium high, and high frequency phonocardiograms, respectively; $3 L$, third left intercostal space; $P$ and $A$, pulmonary and aortic valve components of the second heart sound, respectively; ECG, electrocardiogram.

Keywords: added sound; abnormal relaxation; hyper-tensive heart disease; echocardiography

Added (extra) sounds during early diastole? include a mitral opening sound and a pericar- - V dial knocking sound, as well as a third heart sound. However, it is very rare to find these 8 heart sounds during the isovolumic relaxationo period. ${ }^{12}$ We report here a patient with hypertensive heart disease, left ventricular systolic dysfunction, and complete left bundle branch $\stackrel{2}{=}$ block in whom an added sound was heard $\Im$ during the isovolumic relaxation period.

\section{Case report}

The patient was a 69 year old woman who had been treated for hypertension by her familyo doctor. In May 1995, she began to experience precordial chest pressure and she was treated with sublingual glyceryl trinitrate. When this윽 symptom became frequent and was accompanied by dyspnoea, she again consulted hero doctor and a diagnosis of congestive heart failure was made, based on a finding of pulmonary congestion on a chest radiograph. She was referred to our hospital for further investigation.

Physical examination showed a heart rate of 88 beats $/ \mathrm{min}$ and regular, and blood pressure of $170 / 78 \mathrm{~mm} \mathrm{Hg}$. On auscultation, reversed? splitting of the second heart sound and an added sound during early diastole, similar to $\mathrm{a}$ third heart sound, were heard at the cardiaco apex. A systolic ejection murmur, grade $2 / 6$ by the Levine classification, was also heard at the left sternal border in the third and fourth inter-o costal spaces. The liver was not palpable, and no lower extremity oedema was noted. The heart appeared enlarged, with a cardiothoracicen ratio of $62 \%$, and pulmonary congestion wasto noted on chest radiograph. Electrocardiogram showed normal sinus rhythm with slightly pro-尺 longed PR interval $(200 \mathrm{~ms})$, poor $R$ wave $\stackrel{\mathbb{Q}}{\Omega}$ progression in leads V1-V3, and complete lefto bundle branch block with left axis deviation $\left(-40^{\circ}\right)$ and a prolonged QRS intervafo (150 ms).

Phonocardiography showed a decrease in the first heart sound, reversed splitting of the second heart sound, and a low pitched added sound at the cardiac apex during early diastole (fig 1). This added sound did not change dur- 
ing respiration. A systolic ejection murmur, consistent with Levine grade $2 / 6$, also was recorded from the cardiac apex to the left sternal border in the third intercostal space.

An $M$ mode echocardiogram showed left ventricular enlargement (end diastolic diameter $7 \cdot 0 \mathrm{~cm}$, end systolic diameter $5 \cdot 7 \mathrm{~cm}$ ), a decrease in percent fractional shortening of the left ventricle $(23 \%)$, and left atrial enlargement $(5.0 \mathrm{~cm})$. There was no evidence of heart valve or pericardial disease. On simultaneous recordings using phonocardiogram, $M$ mode, and pulsed Doppler echocardiogram, this added sound coincided with a sudden interruption of the early diastolic motion of

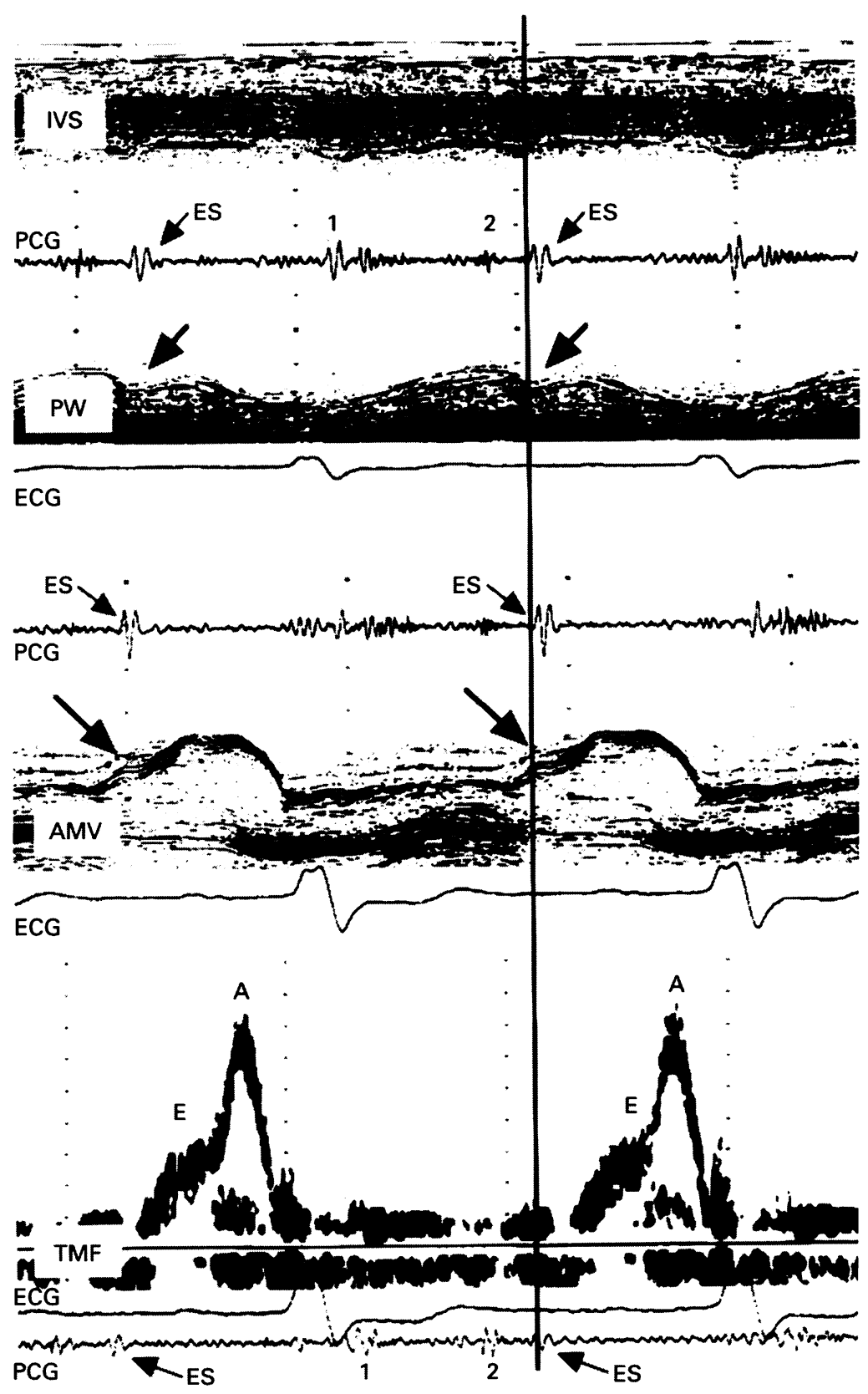

Figure 2 Simultaneous recordings of phonocardiogram, $M$ mode, and pulsed Doppler echocardiograms. The extra sound (ES) coincides with a sudden interruption (arrows) of the early diastolic motion of the left ventricular posterior wall (PW) (top), the initial phase of abnormal forward movement (arrows) during early diastole of the anterior mitral valve (AMV) (middle), and just before the early diastolic flow (E) of the transmitral flow velocity (TMF) (bottom). IVS, interventricular septum; 1 , the first heart sound; 2 , the second heart sound; PCG, phonocardiogram; $A$, atrial systolic wave of the transmitral flow velocity; ECG, electrocardiogram. the left ventricular posterior wall and an initial phase of abnormal forward movement of the anterior leaflet of the mitral valve during early diastole, and came just before the early diastolic transmitral flow, consistent with the isovolumic relaxation period (fig 2 ).

Pulsed Doppler tissue imaging showed that the early diastolic motion velocity from the base to the middle of the left ventricular posterior wall was markedly high and sharp, and its peak coincided with the added sound (fig 3, top). A coronary angiogram showed a $50 \%$ stenosis of the circumflex branch of the left coronary artery. On left ventriculography there was diffuse hypokinesis of the left ventricular wall with a left ventricular ejection fraction of $45 \%$ and abnormal inward movement of the left ventricular posterior wall during early diastole, as detected by $M$ mode echocardiography. The left ventricular pressure curve showed a gradual decline from the isovolumic relaxation period to mid-diastole, indicating a left ventricular relaxation abnormality, and a notch during early diastole, probably corresponding to the occurrence of the added sound (fig 3, bottom). The left ventricular end diastolic pressure was $17 \mathrm{~mm} \mathrm{Hg}$. During her hospital admission, the patient's symptoms and pulmonary congestion were improved with diuretics and vasodilators, but the added sound did not disappear on follow up examinations.

\section{Discussion}

Added sounds during early diastole have been reported in patients with various types of heart disease. ${ }^{1-9}$ The third heart sound and mitral opening sound are representative examples of such sounds. Added sounds are very rare in patients without heart valve disease or pericardial disease, and are most likely to occur with dilated failing hearts. ${ }^{1}$ In the patient described here, this sound occurred before the opening of the mitral valve, that is, during the isovolumic relaxation period, and was distinct from the third heart sound or mitral opening sound.

Added sounds during the isovolumic relaxation period may be caused by abnormal blood flow ${ }^{1}$ or by extracardiac factors. ${ }^{3-5}$ Lee $e t$ $a l^{1}$ have reported a patient with an added sound caused by intraventricular blood flow during the isovolumic relaxation period, and speculated that the mechanism for this sound was a prolongation of the isovolumic relaxation period with asynchronous relaxation. Sasson $e t \mathrm{al}^{2}$ proposed that asynchronous contraction of the left ventricular wall at end systole may result in regional pressure differences within the ventricular cavity during early diastole, and thus lead to intraventricular blood flow during the isovolumic relaxation period and produce added sounds. Our patient had impaired left ventricular systolic function, similar to that reported by Lee et al, ${ }^{1}$ with prolongation of the isovolumic relaxation period accompanied by a curious pattern of left ventricular posterior wall motion and an abnormal pressure curve. However, no distinct intraventricular blood flow signal was detected during 

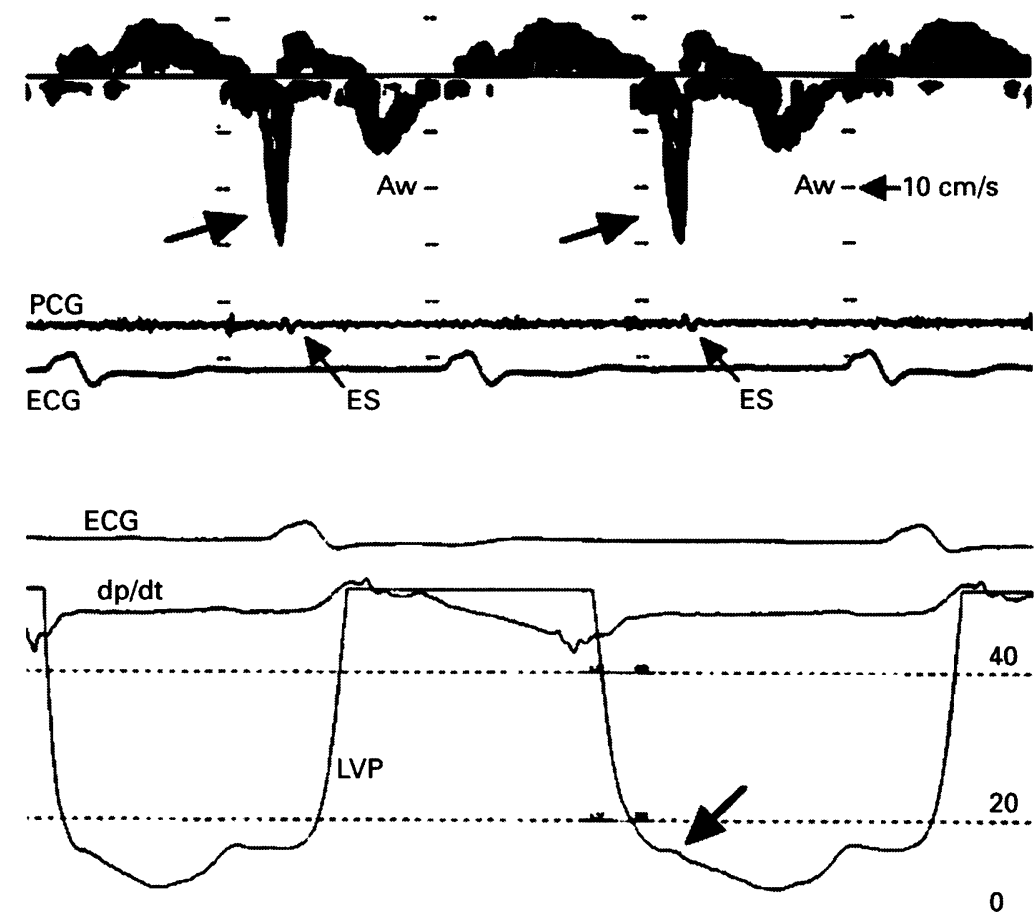

( $\mathrm{mm} \mathrm{Hg})$

Figure 3 Simultaneous recording of phonocardiogram and pulsed Doppler tissue imaging (top), and left ventricular pressure (LVP) curve (bottom). The trace of pulsed Doppler tissue imaging represents velocities of minor axis endocardial motion from the left ventricular posterior wall, and its downward deflection represents outward motion. The early diastolic motion velocity (upper, arrows) at the base of the posterior wall is markedly high and sharp, and its peak coincides with the extra sound (ES). A gradual decline was observed in the LVP curve from the isovolumic relaxation period to the mid-diastole, and a notch (bottom, arrow) was recorded during early diastole. PCG, phonocardiogram; ECG, electrocardiogram; Aw, atrial systolic wave of the posterior wall motion velocity; dp/dt, first derivative of the LVP curve.

the phase corresponding to the added sound, even on detailed examination using pulsed and colour Doppler imaging.

Bonner et $a l^{3}$ have reported an added sound in a patient with a pericardial effusion, and have postulated that this sound was produced by impact between the heart and the pericardium or chest wall during early diastole, resulting from pendular motion due to the pericardial effusion. Ichiyasu et $\mathrm{al}^{4}$ also have reported that left ventricular deviation caused by marked elevation of the diaphragm generated added sounds associated with extracardiac structures in a patient with massive ascites. These reports suggest that these added sounds are caused by impact between an enlarged or deviated heart and the extracardiac structures. In our patient, the area of the left ventricle which is adjacent to the chest wall and diaphragm is presumed to be larger than normal because of marked cardiomegaly. The motion velocity of the left ventricular posterior wall was markedly large and sharp during early diastole, and its peak coincided with the added sound. In addition, the absence of intraventricular blood flow during the isovolumic relaxation period suggests that in this case the added sound was also caused by extracardiac factors.

Rahko et $a l^{10}$ and Shaver et $a l^{11}$ have stated that left ventricular systolic function is more impaired when dilated cardiomyopathy is? accompanied by complete left bundle branch $\overrightarrow{=}$ block. Furthermore, Xiao et $a^{12}$ have reported that prolongation of both the isovolumic contraction period and the isovolumic relaxation period shortens the diastolic period, thus impairing left ventricular filling. Since our patient also had a dilated failing heart, the complication of complete left bundle branch $\overrightarrow{0}$ block may have greatly influenced left ventricu- $\overrightarrow{-}$ lar haemodynamics and produced wall motion abnormalities. Therefore, the occurrence of added sounds may be influenced by atrioven-tricular conductance disturbances in addition $n_{\omega}^{-}$ to the ventricular relaxation abnormality.

In summary, we described a case witho hypertensive heart disease producing unusualo motion of the left ventricular posterior walland a loud added sound during the isovolumic relaxation period. This sound may have been produced by impact between the dilated fail ing heart, with a ventricular relaxation abnor- $\frac{c}{\emptyset}$ mality, and the extracardiac structures duringthe isovolumic relaxation period.

1 Lee $\mathrm{CH}$, Gibson DG. Isovolumic relaxation sound: a new⿳亠二口犬 class of added heart sound? Br Heart $\mathcal{F}$ 1991;65:357-9.

2 Sasson Z, Hatle L, Appleton CP, Jewett M, Alderman EL Popp RL. Intraventricular flow during isovolumic relaxation: description and characterization by Doppler echocardiography. $f \mathrm{Am}$ Coll Cardiol 1987;10:539-46.

3 Bonner AJ, Estevez CM, Dillon JC, Noble RJ, Tavel ME An unusual precordial pulse and sound associated with large pericardial effusion. Chest 1975;68:829-32.

4 Ichiyasu $H$, Nabeyama $S$, Takasugi $M$, Nakashima $Y_{Y}$ Kuroiwa A. Pseudoknock sound in a patient with nephrotic syndrome and massive ascites. Fpn Heart F⿱ nephrotic syndrome

5 Sakamoto $\mathrm{T}$, Ookubo $\mathrm{S}$, Yoshikawa J, Inoue $\mathrm{K}$, Ito Hayashi T. Unusual diastolic heart beat in pericardia? effusion. Fpn Heart f 1972;13:379-93.

6 Bonner AJ, Noble RJ, Feigenbaum H, Tavel ME. Earlȳ diastolic sound associated with mitral valve prolapse Arch Intern Med 1976;136:347-9.

7 Wei JY, Fortuin NJ. Diastolic sounds and murmurs associated with mitral valve prolapse. Circulation 1981;3음 559-64.

8 Cox WR, Damore S, Rubal BJ, Murgo JP. Left atrial myxo oma: phonocardiographic, echocardiographic, andE micromanometric hemodynamic correlations. South Med đo

9 Humen DP, Boughner DR, Gulambusein S, Klein GJ Guiraudon GM. Marked paradoxical septal motion associated with an early diastolic heart sound. Chest $1984 \mathrm{~W}$
86:90-4.

10 Rahko PS, Shaver JA, Salerni R. Evaluation of mechanica events and systolic function dilated cardiomyopathy comparison between patients with and without left bun dle branch block. Acta Cardiol 1988;43:178-84

11 Shaver JA, Rahko PS, Grines CL, Boudoulas H, Wooley CF. Effect of left bundle branch block on the events of the cardiac cycle. Acta Cardiol 1988;43:459-67.

12 Xiao HB, Lee CH, Gibson DG. Effect of left bundle branch block on diastolic function in dilated cardiomys opathy. Br Heart f 1991;66:443-7. 